\title{
Pulmonary mycotic pseudo-aneurysm with a prior history of ventricular septal defect. Case report with review of literature
}

The authors declare no finacial disclosure

\begin{abstract}
In general aneurysms of the pulmonary arteries are less frequent than intracranial, aortic or other vascular locations. Infectious causes include bacteria such as Staphylococcus sp and Streptococcus sp, mycobacteria, Treponema pallidium (syphilis) and rarely fungi. We report a 7 year old female with two right-sided parahilar pseudo-aneurysm of fungal origin with a prior history of ventricular septal defect. Pulmonary mycotic pseudo-aneurysms are very rare and require a high suspicion to diagnose. If a patient is still symptomatic for fever and cough for a long time, and consolidation on x-ray is not improving on antibiotics, contrast-enhanced computed tomography is indicated. It can be suspected that the "friable mass attached to ventricular septal defect patch" was a source of fungeal emboli to pulmonary arteries thus giving weight to the infective endocarditis etiology. A prior history of ventricular septal defect repair could favour fungal endocarditis.
\end{abstract}

Key words: mycotic pseudo-aneurysm, aneurysm of pulmonary artery, ventricular septal defect, fungi emboli, Candida spp.

\section{Introduction}

In general, aneurysms of the pulmonary arteries are less frequent than intracranial, aortic or other vascular locations [1]. Aneurysms of the intrapulmonary arteries can be due to a variety of causes ranging from congenital through infectious or vascular to some iatrogenic in origin [2]. Infectious causes include bacteria such as Staphylococcus and Streptococcus, mycobacteria, Treponema pallidium (syphilis) and rarely fungi [3]. Fungal infections of Aspergillus and candidal origin have been reported [4]. We report a 7 year old female with two right-sided parahilar pseudo-aneurysms of fungal origin with a prior history of ventricular septal defect.

\section{Case report}

A 7 year old female admitted to our hospital with moderate to high grade fever associated with shivers and stiffness for 30 days and also mild cough for 15 days. The patient was operated for ventricular septal defect in the $22^{\text {nd }}$ day of life. Since then she has had no episodes of cyanosis or dyspnea. Growth of the child was appropriate to age. Her past medical history has not been remarkable except for two episodes of pneumonia when she was 3 and 4 years old. There was no history of rash or bleeding. There was no history of breathlessness or vomiting. She was admitted to a private hospital 10 days before and the fever had subsided because of administration of antipyretic medications then. On examination she was febrile. Blood pressure and pulse were within normal limits. Respiratory examination showed decreased breath sounds on the right side. Cardiac examination showed an ejection systolic murmur but no organomegaly. Hematological profile of the patient was almost unremarkable.

Chest X-ray was done which showed well -defined opaque lesion seen in the right parahilar

Address for correspondence: Rajaram Sharma, Department of Radiology, Seth GS medical college and KEM Hospital, India, e-mail: rajaramsharma12345@gmail.com 10.5603/PiAP.2016.0021

Received: 17.10.2015

Copyright (C) 2016 PTChP

ISSN 0867-7077 

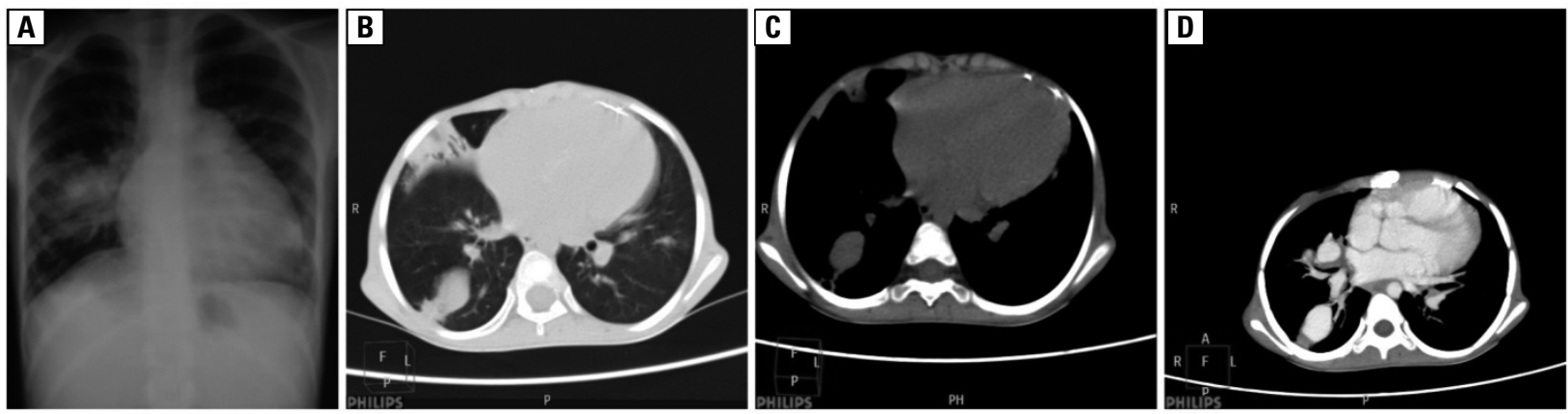

Figure 1. A - plain radiograph of chest showing a well-defined opaque lesion seen in the right para hilar region. The upper lateral margin of the descending right pulmonary artery is silhouetting with the lesion. There is ill defined haziness seen in left lower zone and costo-phrenic angle. Mild cardiomegaly is also evident; B - axial image of high resolution computed tomography of the chest showing a well-defined oval lesion in the posterior segment of right lower lobe. There is pleural thickening distal to it. There is consolidation noted in lateral segment of right middle lobe; $\mathbf{C}-$ axial image of computed tomography scan of the chest in soft tissue window showing a well-defined oval lesion in the posterior segment of right lower lobe. It is homogenous in density and there is pleural thickening distal to it. There is consolidation noted in lateral segment of right middle lobe; $\mathbf{D}$ - post contrast axial image of computed tomography scan of the chest showing that the lesion is continuous with segmental branch of pulmonary artery and enhancing similar to it. These features are suggestive of pseudoaneurysm. There is another pseudoaneurysm seen in the right para hilar region

region. The upper lateral margin of the descending right pulmonary artery is silhouetting with the lesion. There was ill defined haziness seen in left lower zone and costo-phrenic angle. Mild cardiomegaly was also evident (Fig. 1A). Following the chest X- ray, computed tomography scan was done. It showed a well-defined oval lesion in the posterior segment of right lower lobe, which was homogenous in density and there was pleural thickening distal to it. There was consolidation noted in lateral segment of right middle lobe (Figs 1B, C). In post-contrast scan, the lesion is continuous with segmental branch of pulmonary artery, which suggest pseudo-aneurysm (Fig. 1D). There was another pseudo-aneurysm seen in the right parahilar region (Figs 2A, B). Patient was transferred to intensive care unit. Two dimensional echocardiography showed mild to moderate leak across the ventricular septal defect patch with a friable mass attached to it $(8 \mathrm{~mm} \times 8 \mathrm{~mm})$ with mild-to-moderate tricuspid regurgitation and moderate pulmonary hypertension. 21-days empirical therapy by intravenous cephalosporin was unsuccessfull. Finally, blood culture revealed growth of Candida species.

\section{Discussion}

Pulmonary artery aneurysms are usually associated with congenital abnormalities whereas acquired causes are associated with pulmonary hypertension and infections, including tuberculosis, syphilis, and endocarditis. There is a huge decrease in pulmonary artey aneurysm associated with infections due to their prompt treatment. However its asscoiation with traumatic events, including catheterization procedures, and in some cases with chronic lung diseases, such as cavitary pulmonary disease and bronchiectasis on the rise [5].

In our case, the etiology was certailnly infectious as there was no history of trauma, and the patient presented with fever and cough. Our patient underwent ventricular septal defect repair in the past, however there was no previous diagnoses of any congenital malformations of pulmonary arteries. It can be suspected that the „friable mass attached to ventricular septal defect" source of fungi emboli to pulmonary arteries thus giving weight to the infective endocarditis etiology. A prior history of ventricular septal defect repair could favour fungal endocarditis.

Pseudo-aneurysms are formed when there is a breach in all the layers of the blood vessel and extravasated blood is contained within the surrounding clot. Pseudo-aneurysms are usually distinct from aneurysms by absence of an endothelium lining [6]. It usually presents with constitutional symptoms like fever and cough. Sometimes hemoptysis is also reported [7].

The lesion is usually formed by of the pulmonary artery from the surrounding focus of infection or direct invasion of the pulmonary vessel wall from intraluminal septic emboli [8]. Radiographically, it presents as focal areas of consolidation or as ill defined nodules, which are indistinguishable from other infectious causes. Sometimes when there is rapid change in contour of a nodule it is suggestive of a mycotic aneurysm [3, 9].

Computed tomography and magnetic resonance imaging have replaced pulmonary angio- 

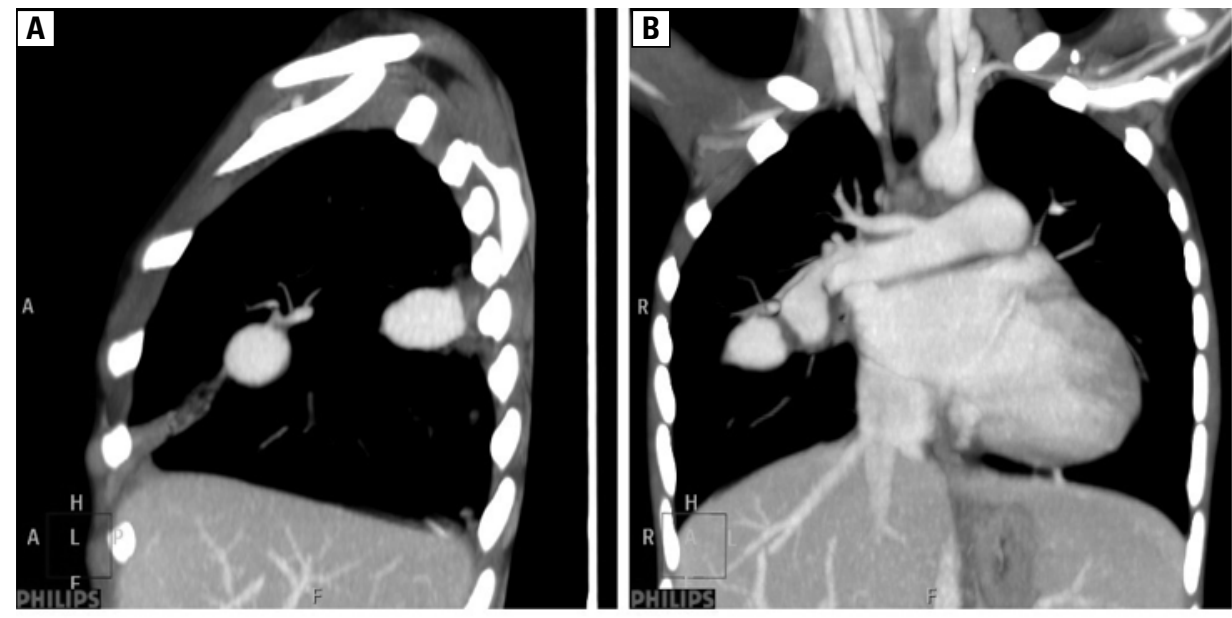

Figure 2. A - post contrast sagittal reconstructed image of computed tomography scan of the chest showing two well defined pseudo-aneurysms along the major fissure in right lobe. There is pleural thickening distal to the pseudoaneurysms; $\mathbf{B}$ - post contrast coronal reconstructed image of computed tomraphy scan of the chest showing two well defined pseudoaneurysms in right para hilar region

graphy as gold standard for diagnosis of this entity. On contrast, enhanced computed tomography, pseudo-aneurysm appears as an enhancing lesion to the central pulmonary artery surrounding/ adjacent to a vessel. Even smaller pseudo-aneurysm can be diagnosed on computed tomography. Angiography shows contained extravasation of contrast and is therefore a definite modality [3, 7].

The experience in management is poor, because diagnosis of mycotic aneurysm is very rare.Transcatheter embolization (treatment of choice), thrombin injection, emergency surgical ligation and lobectomy can all be used in management of pseudo-aneurysms. The type of management usually depends on size of pseudo-aneurysm and vitals of patient [3, 7]. Bigger pseudo-aneurysm can bleed whereas smaller tend to heal by fibrosis on its own [10]. Spontaneous resolution is very rarely reported [11].

\section{Conclusion}

Pulmonary mycotic pseudo-aneurysms are very rare and require a high suspicion to diagnose. If a patient is still symptomatic for fever and cough for a long time and consolidation on chest $\mathrm{x}$-ray is not improving on antibiotics, contrast-enhanced computed tomography is indicated. In our case the etiology was certailnly infectious as there was no history of trauma, and the patient presented with fever and cough. Our patient underwent ventricular septal defect repair in the past, however there was no previous diagnoses of any congenital malformations of pulmonary arteries. In contrast-enhanced computed tomography, pseudo-aneurysm appears as an enhancing lesion to the central pulmonary artery surrounding/ adjacent to a vessel as in our case. Blood culture revealing grow of Candida species points to the fungal etiology. It can be suspected that the „friable mass attached to ventricular septal patch" was a source of fungi emboli to pulmonary arteries thus giving weight to the infective endocarditis etiology. A prior history of ventricular septal defect repair could favour fungal endocarditis.

\section{Conflict of interest}

The authors declare no conflict of interest.

\section{References:}

1. Chung CW, Doherty JU, Kotler R, Finkelstein A, Dresdale A. Pulmonary artery aneurysm presenting as a lung mass. Chest 1995; 108: 1164-1166.

2. Boyd KD, Thomas SJ, Gold J, Boyd AD. A prospective study of complications of pulmonary artery catheterizations in 500 consecutive patients. Chest 1983; 84: 245-249.

3. Kim HS, Oh Y-W, Noh HJ, Lee KY, Kang E-Y, Lee SY. Mycotic Pulmonary Artery Aneurysm as an Unusual Complication of Thoracic Actinomycosis. Korean J Radiol 2004; 5: 68-71. doi:10.3348/kjr.2004.5.1.68.

4. Remy J, Smith M, Lemaitre L, Marache P, Fournier E. Treatment of massive hemoptysis by occlusion of a Rasmussen aneurysm. Am J Roentgenol 1980; 135: 605-606.

5. Sbano H, Mitchell AW, Ind PW, Jackson JE. Peripheral pulmonary artery pseudoaneurysms and massive hemoptysis. Am J Roentgenol 2005; 184: 1253-1259.

6. You CK, Whatley GS. Swan-Ganz catheter-induced pulmonary artery pseudoaneurysm: A case of complete resolution without intervention. Can J Surg 1994; 37: 420-424.

7. Goel S, Kumar A, Gamanagatti S, Gupta A. Spontaneous resolution of post-traumatic pulmonary artery pseudoaneurysm: Report of two cases. Lung India 2013; 30: 203-205. doi: 10.4103/0970-2113.116262.

8. Bartter T, Irwin RS, Nash G. Aneurysms of the pulmonary arteries. Chest 1988; 94: 1065-1075.

9. Jaffe RB, Condon VR. Mycotic aneurysm of the pulmonary artery and aorta. Radiology 1975; 116: 291-298.

10. Symbas PN, Scott HW, Jr Traumatic aneurysm of the pulmonary artery. J Thorac Cardiovasc Surg 1963; 45: 645-649.

11. Rai VK, Malireddy K, Dearmond D, Myers J, Dent DL. Traumatic pseudoaneurysm of the pulmonary artery. J Trauma 2010 69: 730. doi: 10.1097/TA.0b013e3181e7dfac. 